\title{
INVESTIGACIONES CON CFD APLICADAS A BOMBAS CENTRÍFUGAS.
}

\section{INVESTIGATIONS WITH CFD APPLIED TO CENTRIFUGAL PUMPS.}

\author{
Jhan Piero Rojas Suarez ${ }^{1}$ \\ Mawency Vergel Ortega² \\ "Sofia Orjuela Abril' \\ Universidad Francisco de Paula Santander, \\ Cúcuta, Colombia
}

\section{RESUMEN}

En el presente estudio se realiza un estudio de las investigaciones mediante CFD aplicadas a las bombas centrifugas. EI estudio abarca los modelos de turbulencia generalmente utilizado para el estudio de las bombas centrifugas, el procedimiento normalmente usado para el análisis de proceso de optimización y las metodologías avanzadas para identificar los parámetros

$1 \quad$ Facultad de Ingenierías, Universidad Francisco de Paula Santander, Cúcuta, Colombia. Correo jhanpierorojas@ufps.edu.co ORCID iD: https://orcid.org/0000-00032682-9880

2 Departamento de Matemáticas y Estadística, Universidad Francisco de Paula Santander, Norte de Santander, Cúcuta, Colombia, correo: mawencyvergel@ufps.edu.co Orcid: https://orcid.org/0000-0001-8285-2968

$3 \quad$ Facultad de Ciencias Empresariales, Universidad Francisco de Paula Santander, Cúcuta, Colombia. Correo: sofiaorjuela@ufps.edu.co ORCID: https://orcid.org/00000002-9742-8673 geométricos que inciden en el rendimiento. A partir del análisis documental se demuestra que avances en los modelos CFD y la disponibilidad de software, han posibilitado el desarrollo de complejos estudios enfocados en las bombas centrífugas. Los modelos de turbulencia permiten predecir el comportamiento inestable del flujo en las bombas centrifugas. Entre los diferentes modelos disponibles, se destaca el modelo $\mathrm{k}$ -e debido a su capacidad de predicción sin requerir una alta potencia computacional. El proceso de optimización de una bomba centrifuga involucra cambios geométricos que abarcan variaciones en el alabe, difusor, impeler y diámetros de la bomba. El procedimiento general para el análisis de optimización aplicados en bombas centrifugas 
puede ser una base para la construcción de una metodología más rápida y sistemática.

\section{PALABRAS CLAVE:}

Bomba centrifuga, CFD, rendimiento, modelo de turbulencia, optimización.

\section{ABSTRACT}

In the present study a survey of CFD investigations applied to centrifugal pumps is carried out. The study covers the turbulence models generally used for the study of centrifugal pumps, the procedure normally used for the analysis of the optimization process and the advanced methodologies to identify the geometric parameters that affect the performance. From the documentary analysis it is shown that advances in CFD models and the availability of software have made possible the development of complex studies focused on centrifugal pumps. Turbulence models allow predicting the unsteady flow behavior in centrifugal pumps. Among the different models available, the $\mathrm{k}$-e model stands out due to its prediction capability without requiring high computational power. The optimization process of a centrifugal pump involves geometrical changes involving variations in the vane, diffuser, impeller and pump diameters. The general procedure for optimization analysis applied to centrifugal pumps can be a basis for the construction of a faster and more systematic methodology.

\section{KEY WORDS:}

Centrifugal pump, CFD, performance, turbulence model, optimization.

\section{INTRODUCCION}

En la actualidad el proceso de diseño de las bombas centrifugas es de gran importancia debido a las múltiples aplicaciones que tiene en el sector industrial y comercial. Los informes publicados por la comisión europea muestran que aproximadamente un $22 \%$ de la energía producida por motores eléctricos es consumida por los sistemas de bombeo (Meng et al., 2017). Por lo tanto, se hace necesario comprender de forma profunda los factores que intervienen en el desempeño, con el fin de obtener un mejor rendimiento energético y económico.

El proceso de optimización en una bomba centrifuga se puede lograr mediante el estudio de modelos teóricos y/o métodos experimentales. Sin embargo, los modelos teóricos no permiten predecir de forma exacta el comportamiento real, dando como resultado una limitación para el proceso de diseño. En el caso de los métodos experimentales, se presenta las desventajas del alto costo económico y tiempo que es necesario invertir, lo cual resulta poco a tractivo (Olszewski, 2016).

El método más relevante en los últimos años ha sido la aplicación de la dinámica de fluidos Computacional (CFD). Este enfoque aplicación de métodos numéricos por medio de ordenadores de alto rendimiento, contribuyendo en gran medida a la simplicidad de las soluciones en 3D (Ouchbel et al., 2014). En la actualidad la disponibilidad de computadores con más capacidad de procesamiento, el avance en la exactitud y precisión, permite que el análisis mediante CFD se ha competitivo en las industrias donde se buscan de forma constante la construcción de diseño óptimos y el análisis del comportamiento del fluido (Pei et al., 2014).

El interés creciente por la optimización energética en bombas centrifugas ha permitido el desarrollo de técnicas computacionales implementado a análisis paramétricos. (Chen et al., 2017) diseñaron y experimentaron los impulsores de una bomba centrífuga con álabes de estrada retorcidos, estudiando la influencia que tuvo la parametrización en el rendimiento de la bomba. (Zhu et al., 2016) estudió la influencia 
de la altura de la paleta de un difusor de bomba centrífuga en su rendimiento dinámico. (Hou et al., 2016) investigó mediante el análisis CFD los efectos del difusor radial sobre el rendimiento hidráulico de una bomba de petróleo crudo, basados en el método RANS modelaron cuatro tipos de difusores radiales: de doble arco, triple arco, línea en espiral equiangular y línea en espiral variable, obteniendo el mejor rendimiento usando el tercer diseño mencionado. De manera similar, (Ayad et al., 2015) evaluó la influencia de la variación del ancho de la holgura lateral de un impulsor semiabierto en el rendimiento de una bomba centrífuga a diferentes tasas de flujo. Las simulaciones fueron validadas con resultados experimentales y se demostró que la holgura lateral tiene un gran efecto significativo sobre el rendimiento de la bomba centrífuga.

En la presente investigación se realiza un análisis exhaustivo de las investigaciones enfocadas en el uso del CFD aplicado a bombas centrifugas. El objetivo de la investigación es identificar las actuales herramientas utilizadas para el desarrollo de los procesos de optimización en las bombas centrifugas, incluyendo los principales modelos de turbulencia y las metodologías para la identificación de parámetros geométricos claves en el diseño de bombas.

\section{MODELO DE DINÁMICA DE FLUIDOS COMPUTACIONAL PARA BOMBA CENTRÍFUGA}

La compleja geometría de las bombas centrifugas obliga a considerar distintos parámetros geométricos, lo cual se convierte en un desafió para predecir de forma exacta el rendimiento de la bomba. Debido a lo anterior, se ha optado por el uso de métodos de dinámica de fluidos computacional (CFD) para estimar el rendimiento de las bombas. Mediante el análisis CFD es posible obtener una gran cantidad de información relacionado con el campo de flujo, lo cual ha conllevado a que muchos investigadores opten por el uso del CFD como herramienta para el diseño y mejora de las bombas centrifugas.

El método CFD consiste en la solución de la ecuación de continuidad, la ecuación de Navier-Stokes y una ecuación de cierre, la cual consiste en un modelo de turbulencia. Los modelos de turbulencia normalmente utilizados en el análisis CFD incluyen:

el modelo $k-\varepsilon$, el modelo $k-\omega$, el modelo SST $k-\omega$ (Menter's Shear Stress Transport Turbulence), el modelo RNG $k-\varepsilon$ (Renormalization Group) y el método LES (Large Eddy Simulation).

Actualmente, el modelo $k-\varepsilon$ es el mas utilizado en el campo de la ingeniería (Vergel, Díaz, 1998), ya que se ha demostrado su alta confiabilidad para el estudio de la dinámica de los fluidos en modelos 3D (Tan et al., 2015). El modelo de turbulencia RNG $k-\varepsilon$ tiene la ventaja de predecir la turbulencia causada por el movimiento de cizallamiento. En el caso del modelo SST $k-\omega$ se obtiene una mejor predicción del movimiento del flujo bajo condiciones de contrapresión. Este modelo es normalmente empleado para el análisis del campo de flujo y la altura hidráulica en impeler y difusores. El método LES tiene como base la solución de un modelo numérico exacto de segundo orden combinado con un modelo SGS (subgrid scale). En este grupo se encuentran: el modelo de Smagorinsky (SM), el modelo dinámico de Smagorinsky (DSM) y el modelo dinámico mixto (DMM). Las investigaciones realizadas muestran que el método LES permite determinar con precisión el movimiento turbulento del flujo en bombas centrifugas.

La selección del modelo de turbulencia puede diferir de forma ligera los resultados obtenidos. Por lo tanto, es necesario realizar un proceso de comparación para garantizar que el modelo usado se ha el apropiado de cuerdo a las situaciones particulares de cada 
estudio. En la Tabla 1 se muestra un resumen de los modelos de turbulencia utilizados para el estudio de bombas centrifugas.

Tabla 1: Modelos de turbulencia utilizados en la literatura. Fuente de datos:

\begin{tabular}{lll}
\hline Referencia & Modelo de turbulencia & Descripción de la investigación \\
\hline (Zhang et al., 2016) & LES & $\begin{array}{l}\text { Evaluación de la interacción entre el campo de flujo de } \\
\text { una bomba centrifuga y la inestabilidad del estator para } \\
\text { condiciones de baja velocidad. }\end{array}$ \\
\hline (Zhou et al., 2015) & SST $k-\omega$ & $\begin{array}{l}\text { Comparación entre las predicciones de las simulaciones } \\
\text { CFD y los resultados experimentales para el estudio } \\
\text { del comportamiento del campo de flujo de un difusor en } \\
\text { condiciones de alta carga. }\end{array}$ \\
\hline (Tan et al., 2015) & RNG $k-\varepsilon$ & $\begin{array}{l}\text { Análisis del campo de flujo y la influencia de la cavitación en } \\
\text { condiciones de carga parciales. }\end{array}$ \\
\hline (Kaewnai et al., 2009) & $k-\epsilon$ & $\begin{array}{l}\text { Predicción y análisis del rendimiento del impeler de una } \\
\text { bomba centrifuga de flujo radial. }\end{array}$ \\
\hline
\end{tabular}

Elaborado por los autores.

\section{PROCEDIMIENTO GENERAL DE OPTIMIZACIÓN EN BOMBAS CENTRIFUGAS}

Los cambios en el diseño es una de las principales formas para incrementar el rendimiento de las bombas centrifugas. A lo largo de los últimos años se ha utilizado diferentes técnicas para la optimización mediante CFD. En la Figura 1 se muestra el procedimiento generalmente implementado para la optimización de bombas centrifugas.
El paso 1 consiste en utilizar el diseño de experimentos (DOE) para identificar las variables que se consideran para el rediseño. En el paso 2 se utiliza la herramienta CFD para simular un diseño especifico de bombas. Permitiendo identificar parámetros de eficiencia, cabeza, NPSHr, entre otros. Para el paso 3 se utiliza un modelo sustituto, de esta forma, se establece una relación entre las variables de diseño y los parámetros de optimización. El paso 4 consiste en una verificación del modelo sustituto, mediante la comparación del modelo con los valores predichos en la simulación. 
Figura 1: Procedimiento general de optimización. Fuente: Autores.

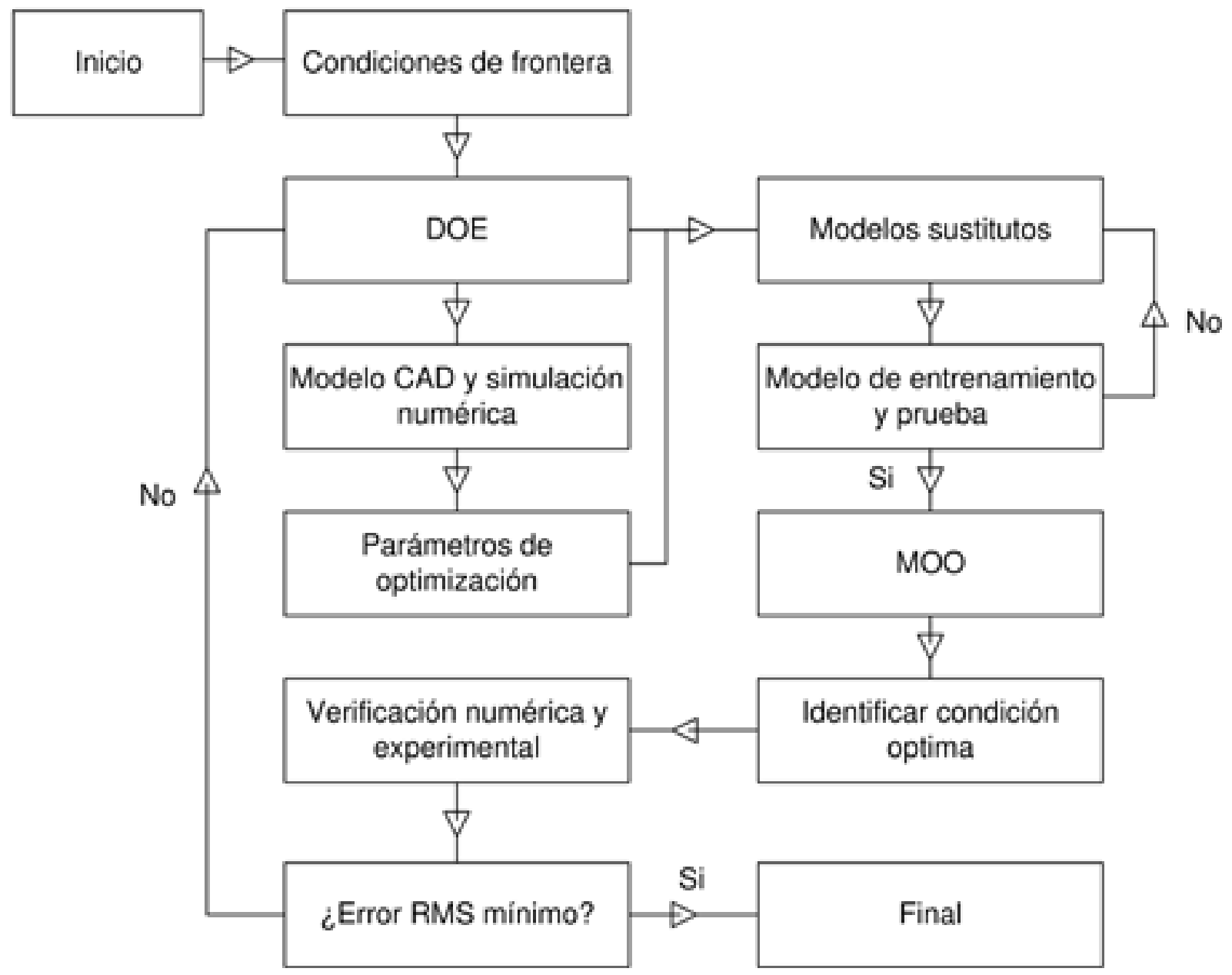

Posteriormente, en el paso 5 se aplica un algoritmo de optimización multiobjetivo (MOO) para encontrar los valores óptimos de las variables de diseño. Finalmente, se identifican los valores óptimos de las variables de diseño (paso 6) y se realiza un proceso de validación mediante simulación y prueba experimental.

\section{DISCUSIÓN DE METODOLOGÍA PARA EL MODELADO CFD}

En la Figura 2 se muestran los parámetros geométricos que normalmente son seleccionados para la optimización de las bombas centrifugas. 
Figura 2: Parámetros geométricos de las bombas centrifugas. Fuente: autores.

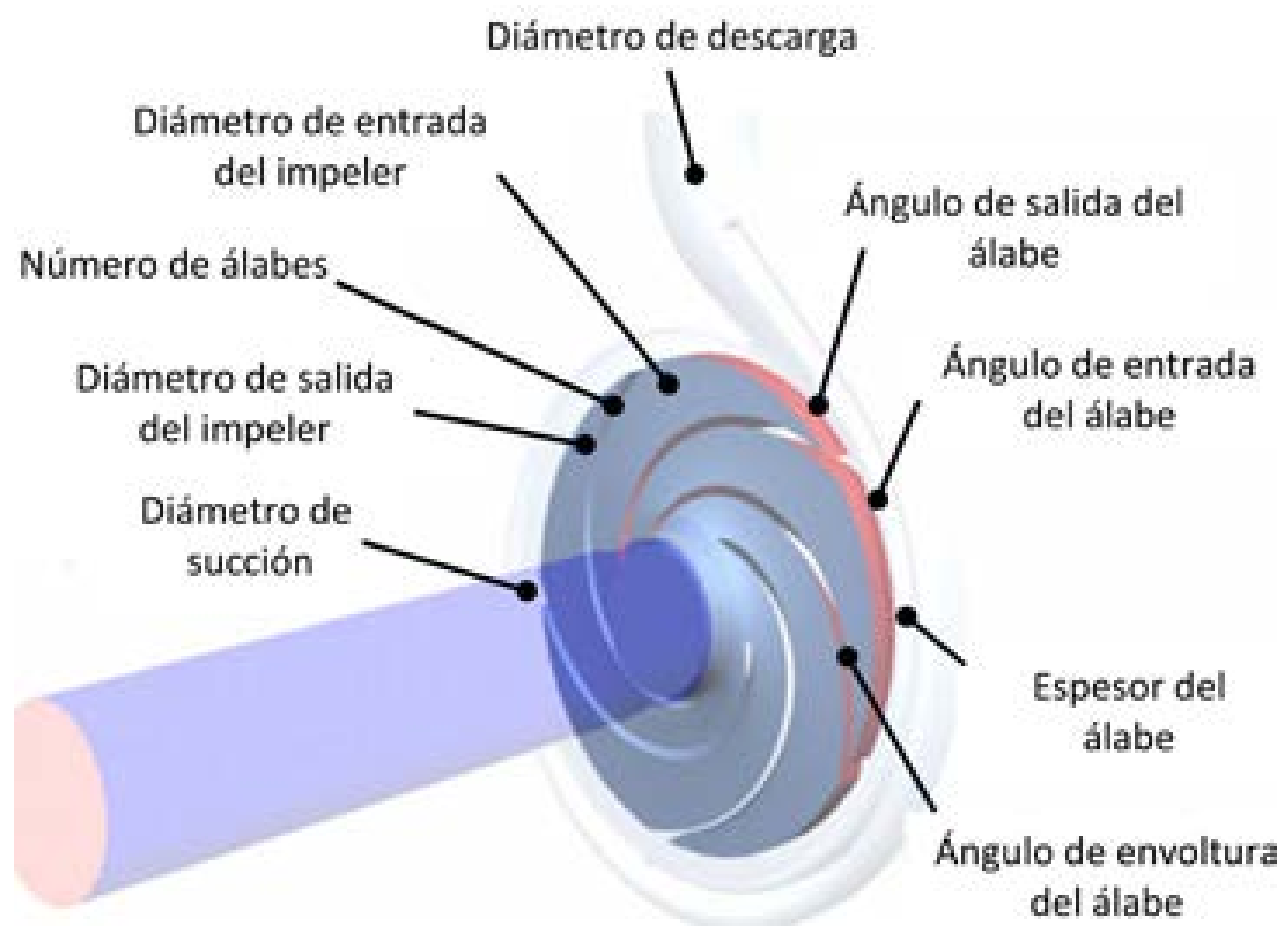

El número de alabes, el ángulo del alabe de entrada y el ángulo del alabe de salida son los principales parámetros que afectan la eficiencia de una bomba centrifuga. Debido a esto, son normalmente seleccionados para la investigación de nuevos diseños. El estudio de (Tao et al., 2018) indicó que la reducción en el ángulo de entrada y el incremento del ángulo de salida de los alabes de la bomba permiten mejorar el rendimiento de bombas utilizadas para el transporte de fluidos viscosos.

(Houlin et al., 2010) concluyó que el aumento en el número de alabes permite la formación de líneas de flujo uniformes e incrementar la altura de la bomba. (Siddique et al., 2018) demostró que el ángulo de salida del alabe es un parámetro clave para el incremento de la velocidad periférica de la bomba. (Tong et al., 2019) realizó un proceso de optimización considerando el ángulo del flujo de salida del alabe y su efecto en las perdidas energéticas. (Liu et al., 2019 utilizó la curva de Bézier y la función lineal para la optimización del perfil del alabe, logrando mejorar la eficiencia hidráulica de la bomba. (Y. Wang \& Huo, 2018) utilizó los parámetros de eficiencia, cabeza y NPSH para el desarrollo de una metodología de optimización.

En la actualidad se utilizan de forma continua combinaciones de métodos para lograr una mejoría en el rendimiento de las bombas centrifugas, entre los cuales se encuentran el DOE, el algoritmo MOO y el modelo sustituto. En la Tabla 2 se muestra un resumen de estudios enfocados en la optimización de bombas centrifugas. 
Tabla 2: Estudio de optimización en bombas centrifugas. Fuente de datos: Elaborado por los autores.

\begin{tabular}{|c|c|c|c|c|}
\hline Referencia & $\begin{array}{l}\text { Modelo } \\
\text { sustituto }\end{array}$ & $\begin{array}{l}\text { Algoritmo de } \\
\text { optimización }\end{array}$ & $\begin{array}{l}\text { Variable de } \\
\text { estudio }\end{array}$ & $\begin{array}{l}\text { Parámetro de } \\
\text { optimización }\end{array}$ \\
\hline $\begin{array}{l}\text { (Tao et al., } \\
2018 \text { ) }\end{array}$ & ANN & MOO & $\begin{array}{l}\text { - Ángulo del } \\
\text { álabe } \\
\text { - Ángulo de } \\
\text { envoltura }\end{array}$ & $\begin{array}{l}\text { - Eficiencia } \\
\text { - Cabeza }\end{array}$ \\
\hline $\begin{array}{l}\text { (Shim et al., } \\
\text { 2018) }\end{array}$ & RSA & MOGA & $\begin{array}{l}\text { - Ángulo de } \\
\text { entrada y } \\
\text { salida del } \\
\text { impeler }\end{array}$ & $\begin{array}{l}\text { - Eficiencia } \\
\text { - NPSHr }\end{array}$ \\
\hline $\begin{array}{l}\text { (Derakhshan \& } \\
\text { Bashiri, 2018) }\end{array}$ & $\begin{array}{l}\text { - ANN } \\
\text { - Red } \\
\text { neuronal } \\
\text { feedforward }\end{array}$ & ES & $\begin{array}{l}\text { - Diámetro de } \\
\text { succión } \\
\text { - Ancho y } \\
\text { diámetro del } \\
\text { impeler } \\
\text { - Ángulo de } \\
\text { entrada y } \\
\text { salida del } \\
\text { álabe }\end{array}$ & $\begin{array}{l}\text { - Eficiencia } \\
\text { - Cabeza }\end{array}$ \\
\hline (An et al., 2016) & $\begin{array}{l}\text { Red neuronal } \\
\text { feedforward }\end{array}$ & NSGA-II & $\begin{array}{l}\text { - Ángulo de } \\
\text { entrada y } \\
\text { salida del } \\
\text { álabe } \\
\text { - Sección del } \\
\text { impeler }\end{array}$ & $\begin{array}{l}\text { - Eficiencia } \\
\text { - NPSHr }\end{array}$ \\
\hline
\end{tabular}

\subsection{DISEÑO DE EXPERIMENTOS}

EI DOE es la base de los procesos de optimación, debido a que permiten determinar la importancia de las variables de diseño e identificar su combinación optima (Pei et al., 2019), lo cual implica una mejora significativa en el rendimiento de la bomba y una reducción en el proceso de rediseño.
Entre los métodos de diseño experimental más comunes se encuentra la matriz ortogonal (OA) y el muestreo de hipercubo latino optimizado (OLHS). Las investigaciones de (Y. Wang \& Huo, 2018)(W. Wang et al., 2019) son una muestra de los estudios que 
han utilizado el método ortogonal para el diseño de bombas centrifugas.

\subsection{ALGORITMO DE OPTIMIZACIÓN MULTIOBJETIVO}

El algoritmo MOO busca identificar un máximo o mínimo de un conjunto de funciones objetivas, las cuales se encuentran bajo restricciones variables. Debido a la dificultad de encontrar una solución óptima que satisfaga todas las funciones objetivas, generalmente, se busca una condición que permita incrementar el rendimiento en comparación con un estado inicial. El funcionamiento del algoritmo se basa en la evolución de parámetros de diseño bases (Liu et al., 2019

Entre los trabajos que han utilizado este tipo de metodologías se encuentra (Yanshu et al., 2012), el cual implementó un algoritmo genético para estimar el rendimiento de una bomba centrifuga. (Safikhani et al., 2011) y (Huang et al., 2015) utilizaron un algoritmo genético multiobjetivo para mejorar el rendimiento de una bomba a través de cambios en el impulsor.

\subsection{MODELO SUSTITUTO}

Los modelos sustitutos permiten la construcción de una función a partir de un conjunto de datos, para posteriormente predecir la función en un nuevo conjunto de puntos. En ingeniería se usa de forma recurrente, ya que es de fácil entrenamiento y mantiene una precisión aceptable. Entre los modelos sustitutos que se han utilizado para la optimización de las bombas centrifugas se encuentran: la red neuronal artificial, el análisis de superficie de respuesta, la red neuronal de base radial y Kriging.

\section{CONCLUSIONES}

En la presente investigación se realizó una revisión de modelo turbulencias, procedimiento y metodologías utilizadas para el desarrollo de procesos de optimización aplicados en bombas centrifugas.

El análisis de la literatura muestra que los avances en los modelos CFD y la disponibilidad de softwares con mayores capacidades de procesamiento ha permitido ampliar la complejidad de los estudios enfocados en las bombas centrífugas. El uso de modelos de turbulencia permite predecir el comportamiento inestable del flujo en las bombas centrifugas, lo cual es de gran importancia para la búsqueda de un mejor rendimiento. Sin embargo, es necesario una adecuada selección y comprobación del modelo de turbulencia, ya que este puede afectar el criterio para definir una condición optima.

En el proceso de optimización de las bombas centrifugas están involucradas un número significativo de variables geométricas, las cuales pueden implicar cambios en los diámetros de entrada y salida de la bomba, cambios geométricos en el impeler y difusor, y variaciones en el número y perfil de los álabes.

En el estudio se describe un procedimiento general para el análisis de optimización aplicados en bombas centrifugas. De esta forma, se busca establecer una base para el desarrollo de una metodología sistemática y rápida que permita predecir los fenómenos de cavitación y optimizar la estructura de las bombas centrifugas. 


\section{REFERENCIAS BIBLIOGRÁFICAS}

An, Z., Zhounian, L., Peng, W., Linlin, C., \& Dazhuan, W. (2016). Multi-objective optimization of a low specific speed centrifugal pump using an evolutionary algorithm. Engineering Optimization, 48(7), 1251-1274. https://doi.org/ https://doi.org/10.1080/030521 5X.2015.1104987

Ayad, A. F., Abdalla, H. M., \& Abo ElAzm, A. S. (2015). Numerical Study of the Semi-Open Centrifugal Pump Impeller Side Clearance. International Conference on Aerospace Sciences and Aviation Technology, 16(AEROSPACE SCIENCES I\& AVIATION TECHNOLOGY, ASAT-16-May 26-28, 2015), 1-14. https://doi. org/10.21608/ASAT.2015.23015

Chen, H., He, J., \& Liu, C. (2017). Design and experiment of the centrifugal pump impellers with twisted inlet vice blades. Journal of Hydrodynamics, Ser. B, 29(6), 1085-1088. https://doi. org/https://doi.org/10.1016/S10016058(16)60822-3

Derakhshan, S., \& Bashiri, M. (2018). Investigation of an efficient shape optimization procedure for centrifugal pump impeller using eagle strategy algorithm and ANN (case study: slurry flow). Structural and Multidisciplinary Optimization, 58(2), 459-473. https:// doi.org/https://doi.org/10.1007/ s00158-018-1897-3

Hou, H. C., Zhang, Y. X., Xu, C., Zhang, J. Y., \& Li, Z. L. (2016). Effects of radial diffuser hydraulic design on a double-suction centrifugal pump. IOP Conference Series: Materials Science and Engineering, 129(1), 12017.

Houlin, L., Yong, W., Shouqi, Y., Minggao, T., \& Kai, W. (2010). Effects of blade number on characteristics of centrifugal pumps. Chinese Journal of Mechanical Engineering-English Edition, 6, 742. https://doi.org/10.3901/ CJME.2010.06.742

Huang, R., Luo, X., Ji, B., Wang, P., Yu, A., Zhai, Z., \& Zhou, J. (2015). Multiobjective optimization of a mixed-flow pump impeller using modified NSGA-II algorithm. Science China Technological Sciences, 58(12), 2122-2130. https:// doi.org/https://doi.org/10.1007/s11431015-5865-5

Kaewnai, S., Chamaoot, M., \& Wongwises, S. (2009). Predicting performance of radial flow type impeller of centrifugal pump using CFD. Journal of Mechanical Science and Technology, 23(6), 1620-1627. https://doi.org/https://doi. org/10.1007/s12206-008-1106-1

Liu, X., Li, H., Shi, X., \& Fu, J. (2019). Application of biharmonic equation in impeller profile optimization design of an aero-centrifugal pump. Engineering Computations, 36(5), 1764-1795. https://doi.org/https://doi.org/10.1108/ EC-08-2018-0378

Meng, F., Zhang, H., Yang, F., Hou, X., Lei, B., Zhang, L., Wu, Y., Wang, J., \& Shi, Z. (2017). Study ofefficiency ofamultistage centrifugal pump used in engine waste heat recovery application. Applied Thermal Engineering, 110, 779-786. 
https://doi.org/https://doi.org/10.1016/j. applthermaleng.2016.08.226

Olszewski, P. (2016). Genetic optimization and experimental verification of complex parallel pumping station with centrifugal pumps. Applied Energy, 178, 527-539. https://doi.org/https://doi.org/10.1016/j. apenergy.2016.06.084

Ouchbel, T., Zouggar, S., Elhafyani, M. L., Seddik, M., Oukili, M., Aziz, A., \& Kadda, F. Z. (2014). Power maximization of an asynchronous wind turbine with a variable speed feeding a centrifugal pump. Energy Conversion and Management, 78, 976-984. https://doi.org/https://doi.org/10.1016/j. enconman.2013.08.063

Pei, J., Gan, X., Wang, W., Yuan, S., \& Tang, Y. (2019). Multi-objective shape optimization on the inlet pipe of a vertical inline pump. Journal of Fluids Engineering, 141(6), 061108. https://doi.org/https://doi. org/10.1115/1.4043056

Pei, J., Yuan, S., Li, X., \& Yuan, J. (2014). Numerical prediction of $3-D$ periodic flow unsteadiness in a centrifugal pump under part-load condition. Journal of Hydrodynamics, 26(2), 257-263. https://doi.org/https://doi.org/10.1016/ S1001-6058(14)60029-9

Safikhani, H., Khalkhali, A., \& Farajpoor, M. (2011). Pareto based multi-objective optimization of centrifugal pumps using CFD, neural networks and genetic algorithms. Engineering Applications of Computational Fluid Mechanics, 5(1), 37-48. https://doi.org/https://doi.org/10
$.1080 / 19942060.2011 .11015351$

Shim, H.-S., Kim, K.-Y., \& Choi, Y.-S. (2018). Three-objective optimization of a centrifugal pump to reduce flow recirculation and cavitation. Journal of Fluids Engineering, 140(9), $091202 . \quad$ https://doi.org/https://doi. org/10.1115/1.4039511

Siddique, M. H., Afzal, A., \& Samad, A. (2018). Design optimization of the centrifugal pumps via low fidelity models. Mathematical Problems in Engineering, 2018. https://doi.org/ https://doi.org/10.1155/2018/3987594

Tan, L., Zhu, B., Wang, Y., Shuliang, C., \& Gui, S. (2015). Numerical study on characteristics of unsteady flow in a centrifugal pump volute at partial load condition. Engineering Computations, 32(6), 1549-1566. https://doi.org/ https://doi.org/10.1108/EC-05-20140109

Tao, R., Xiao, R., Zhu, D., \& Wang, F. (2018). Multi-objective optimization of double suction centrifugal pump. Proceedings of the Institution of Mechanical Engineers, PartC: JournalofMechanical Engineering Science, 232(6), 1108$1117 . \quad$ https://doi.org/https://doi. org/10.1177/0954406217699020

Tong, Z., Cheng, Z., \& Tong, S. (2019). Preliminary design of multistage radial turbines based on rotor loss characteristics under variable operating conditions. Energies, 12(13), 2550. https://doi.org/https://doi.org/10.3390/ en12132550 
Vergel Ortega, M y Diaz Gómez, C. (1998). La base teorica de la simulacion de eventos. revista Respuestas 3(1)

Wang, W., Osman, M. K., Pei, J., Gan, X., \& Yin, T. (2019). Artificial neural networks approach for a multi-objective cavitation optimization design in a double-suction centrifugal pump. Processes, 7(5), 246. https://doi.org/https://doi.org/10.3390/ pr7050246

Wang, Y., \& Huo, X. (2018). Multiobjective optimization design and performance prediction of centrifugal pump based on orthogonal test. Advances in Materials Science and Engineering, 2018. $\quad$ https://doi.org/https://doi. org/10.1155/2018/6218178

Yanshu, Z., Shisha, Z., Dazhou, Z., \& Cheng, $W$. (2012). Predicting performance of centrifugal pump by combining genetic algorithm with BP neural network. Mechanical Science and Technology for Aerospace Engineering, 31(8), 1274-1279.

Zhang, N., Yang, M., Gao, B., Li, Z., \& Ni, D. (2016). Investigation of rotor-stator interaction and flow unsteadiness in a low specific speed centrifugal pump. Strojniški Vestnik-Journal of Mechanical Engineering, 62(1), 21-31. https://doi. org/10.5545/sv-jme.2015.2859

Zhou, L., Shi, W., Cao, W., \& Yang, H. (2015). CFD investigation and PIV validation of flow field in a compact return diffuser under strong part-load conditions. Science China Technological Sciences, 58(3), 405-414. https://doi.org/https:// doi.org/10.1007/s11431-014-5743-6
Zhu, X., Li, G., Jiang, W., \& Fu, L. (2016). Experimental and numerical investigation on application of half vane diffusers for centrifugal pump. International Communications in Heat and Mass Transfer, 79, 114-127. https://doi.org/https://doi.org/10.1016/j. icheatmasstransfer.2016.10.015 